\section{Assessment of functional nasolacrimal duct obstruction - a survey of ophthalmologists in the southwest}

\section{Introduction}

A watering eye is a common problem with many causes. There may be problems with the lacrimal drainage system, ocular surface or the eyelids, and a few patients just seem to make too many tears. The term 'functional nasolacrimal duct obstruction' has been used to describe patients with epiphora with evidence of abnormal tear drainage, but where the nasolacrimal duct is partially or completely patent on syringing. It is not a good term as many older and the majority of younger patients have anatomical abnormalities on dacryocystography. ${ }^{1}$ Use of this term implies exclusion of causes of hypersecretion and lid problems, as well as problems with the part of the lacrimal drainage system proximal to the nasolacrimal duct including punctal stenosis and canalicular block.

There is evidence that selected patients with functional nasolacrimal duct obstruction do well with dacrocystorhinostomy (DCR) operations, with a good chance of symptom improvement or resolution..$^{2-6}$

We observed that some patients with epiphora were being assessed solely by syringing of the lacrimal system. If the drainage system was patent, the problem was assumed to lie elsewhere and the patient was not considered for DCR surgery. We felt that the lack of a standardised workup may mean that patients with functional obstruction are passed over and not considered for surgery.

This was illustrated by a survey of American lacrimal specialists where 138 out of 300 doctors replied to a questionnaire in which they were asked to manage a hypothetical patient who had epiphora, but was patent on syringing. ${ }^{7,8}$
Presented as a poster at the Royal College of Ophthalmologists Annual Congress, May 2002
Keywords: functional nasolacrimal duct obstruction; epiphora investigations are undertaken, so patients with a highly treatable cause of epiphora can be offered dacrocystorhinostomy. Eye (2004) 18, 20-23. doi:10.1038/sj.eye.6700522 
Huge gaps and inconsistencies in the workup of patients were revealed.

We set out to investigate this on a regional level by carrying out a survey of ophthalmologists practising in the southwest.

\section{Materials and methods}

Questionnaires were sent out to 198 hospital-based ophthalmologists of all grades in the southwest of England (Table 1). Enquiries were made about their investigation and management of patients with epiphora. Our initial aim was to establish how often individual doctors were seeing patients with epiphora, and we then asked further questions about their clinical assessment of patients. If assessment revealed a patient with epiphora whose lacrimal system appeared patent to syringing, we asked about whether doctors would request further investigations. Doctors were asked to reply to the questions by circling one of the following replies: always/frequently/sometimes/rarely/never.

\section{Results}

A good response was received with 163 of 198 questionnaires returned (82\%) (Table 2). Eight doctors $(5 \%)$ never assessed patients with epiphora and answered no further questions, 42 doctors (25\%) assessed such patients rarely, and $113(70 \%)$ performed such assessments frequently or sometimes.

Of those doctors answering further questions, 65/158 $(41 \%)$ rarely or never performed a fluorescein dye disappearance test (FDDT). Jones tests were not generally used, with only 18/155 (12\%) carrying them out always or frequently.

Almost all patients were having their lacrimal systems syringed, with 152 (96\%) answering always or frequently, but only in half the cases was syringing carried out by a doctor straightaway. In all, 45/158 (28\%) of patients were having syringing carried out by a nurse and only repeated by a doctor if a problem was detected.

Further questions focused on the subsequent investigation and management of patients with epiphora and lacrimal systems that were patent to syringing (ie appeared to have a functional nasolacrimal duct obstruction). Very few people considered lacrimal scintigraphy with only $6 / 154(3 \%)$ requesting this regularly and $139 / 154(84 \%)$ rarely or never. A few more people used DCGs with 20/158 (13\%) requesting this regularly but still 90/158 (57\%) rarely or never.

\section{Discussion}

Most ophthalmologists assess epiphora. Functional nasolacrimal duct obstruction seems to be an under-recognised problem, largely because of the poor quality of workup of patients with epiphora and lack of use of appropriate tests and investigations.

When a patient presents with painless epiphora, a history and slit-lamp examination are needed to exclude ocular surface disease and lid problems. Following this there are a number of simple and noninvasive tests that could provide a great deal of information about the nature of the problem.

Table 1 Questionnaire

\begin{tabular}{|c|c|c|c|c|c|}
\hline \multirow{2}{*}{$\begin{array}{l}\text { Question } \\
\text { Do you assess patients with epiphora } \\
\text { for DCR surgery? }\end{array}$} & \multicolumn{5}{|l|}{ Answers } \\
\hline & & Frequently & Sometimes & Rarely & Never \\
\hline \multicolumn{6}{|l|}{ In your assessment of patients with epiphora: } \\
\hline Would you do an FDDT? & Always & Frequently & Sometimes & Rarely & Never \\
\hline Would you do a Jones 1 test? & Always & Frequently & Sometimes & Rarely & Never \\
\hline Would you syringe the lacrimal system? & Always & Frequently & Sometimes & Rarely & Never \\
\hline Who usually syringes the patient? & Yourself & A junior doctor & Eye nurse & & \\
\hline $\begin{array}{l}\text { If the tear duct were patent would you arrange } \\
\text { lacrimal scintillography (isotope scan)? }\end{array}$ & Always & Frequently & Sometimes & Rarely & Never \\
\hline $\begin{array}{l}\text { If the tear duct were patent would you arrange } \\
\text { a dacryocystogram? }\end{array}$ & Always & Frequently & Sometimes & Rarely & Never \\
\hline
\end{tabular}


Table 2 Results

\begin{tabular}{|c|c|c|c|c|c|c|c|}
\hline & & Always & Frequently & Sometimes & Rarely & Never & No answer \\
\hline \multirow[t]{2}{*}{ Do you assess patients with epiphora? } & No & & 42 & 71 & 26 & 8 & 15 \\
\hline & $\%$ & & 28 & 48 & 18 & 5 & \\
\hline \multirow[t]{2}{*}{ Would you do an FDDT? } & No & 32 & 22 & 38 & 32 & 33 & 5 \\
\hline & $\%$ & 20 & 14 & 24 & 20 & 21 & \\
\hline \multirow[t]{2}{*}{ Would you do a Jones 1 test? } & No & 9 & 9 & 27 & 49 & 60 & 8 \\
\hline & $\%$ & 6 & 6 & 17 & 32 & 39 & \\
\hline \multirow[t]{3}{*}{ Would you syringe the lacrimal system? } & No & 123 & 29 & 5 & 0 & 0 & 5 \\
\hline & $\%$ & 78 & 18 & 3 & 0 & 0 & \\
\hline & & Self & Junior & Nurse & $\begin{array}{l}\text { Nurse, repeated } \\
\text { if problem }\end{array}$ & & \\
\hline \multirow[t]{3}{*}{ Who syringes the patient? } & No & 79 & 0 & 79 & 44 & & 5 \\
\hline & $\%$ & 50 & 0 & 50 & 28 & & \\
\hline & & Always & Frequently & Sometimes & Rarely & Never & No answer \\
\hline $\begin{array}{l}\text { If the tear duct were patent would you } \\
\text { arrange lacrimal }\end{array}$ & No & 1 & 5 & 18 & 48 & 81 & 9 \\
\hline Scintigraphy? & $\%$ & $<1$ & 3 & 12 & 31 & 53 & \\
\hline \multirow{2}{*}{$\begin{array}{l}\text { If the tear duct were patent would you } \\
\text { arrange a DCG? }\end{array}$} & No & 3 & 17 & 47 & 65 & 25 & 5 \\
\hline & $\%$ & 2 & 11 & 30 & 41 & 16 & \\
\hline
\end{tabular}

An FDDT is probably the single most useful test apart from syringing. It involves placing a drop of $2 \%$ fluorescein in the lower fornix of each eye and leaving for 5 minutes. If the eye remains yellow and the tear meniscus is raised, it suggests that the tear drainage is abnormal. ${ }^{9}$ The test is particularly helpful in unilateral epiphora as the normal side goes white while the abnormal one remains yellow. It is worth remembering that patency cannot be assumed if the dye is gone as there may be an element of washout.

An additional and complementary test is a Jones I test. Dye is applied to the inferior fornix, and looked for in the nose at various intervals. Guzek et $a l^{9}$ suggested that if there is dye detectable in the nose within an appropriate time then this confirms tear hypersecretion, but equally if there is no dye present, obstruction cannot be confirmed. A Jones II test can follow on from this. Excess fluorescein is washed out of the conjunctival sac, topical anaesthetic instilled, and then the lacrimal system irrigated with clear saline, looking for the appearance of fluorescein-stained saline within the nose.

Lacrimal syringing with or without probing is important and sensitive, but an element of skill is required to carry it out, and in inexperienced hands subtleties such as reflux from partial obstruction may be overlooked.

Radiological investigations were considered by very few practitioners in our survey. Dacrocystography is a widely available investigation where radio-opaque dye is injected into the lacrimal system under pressure, and pictures taken. A reasonable anatomical picture is obtained and the investigation can show up any complete anatomical obstruction. Partial obstructions can also be detected in many cases. A limitation of this investigation is that it provides a structural rather than a functional picture. Owing to the high pressure of the dye injection, the passage of the dye through the system is for the most part nonphysiological. It will show an anatomical abnormality in most but not all cases of functional nasolacrimal duct obstruction.

Lacrimal scintigraphy is an alternative radiological investigation. It is designed to give a more physiological picture. A mixture of the tracer and saline is dropped into the lower fornix and pictures taken as the tracer works its way down the lacrimal system. Wearne et $a l^{10}$ compared lacrimal scintigraphy to dacrocystography in cases of functional obstruction, looking at 55 lacrimal systems over a year. They found that $93 \%$ of the DCGs showed an abnormality, compared to $95 \%$ of the lacrimal scintigraphy scans, and the two when used together had a combined sensitivity or $98 \%$. They were also able to subdivide the lacrimal scans into those showing obstruction at different levels, indicating where the delay was, which may have implications when considering the likelihood of success with surgery. 
Recent evidence has shown that patients with functional nasolacrimal duct obstruction do well with either open or endonasal DCRs. O'Donnell and $S_{\text {Shah }}{ }^{2}$ looked at the success rate of external DCR in patients with functional nasolacrimal duct obstruction. They included patients over 16 years of age who were symptomatic of epiphora with normal slit-lamp and lid examinations and clinically patent lacrimal systems. Patency was assessed with a FDDT, Jones I and II tests, and lacrimal syringing, with dacrocystograms (DCG) in borderline cases. Of the 51 patients in the study, 48 (94\%) had improved symptoms postoperatively with minimal or no epiphora. These results are backed up by other studies. Zaidy et $a l^{3}$ compared results of endonasal vs open DCR in the treatment of epiphora from functional nasolacrimal duct obstruction. Patients were selected following lacrimal syringing performed by an ophthalmologist and either lacrimal scintigraphy or DCG. Reasons for exclusion included previous surgery, an inflammatory cause of obstruction, and canalicular disease. In all, 21 patients underwent endonasal DCR and 25 open DCR, both with canalicular intubation. In the endoscopic group, $85.7 \%$ had symptom reduction at 6 months compared to $100 \%$ of the open group. A similar study compared endonasal surgical DCR with an endonasal laser procedure. ${ }^{4}$ Their patients included a subgroup with functional nasolacrimal duct obstruction. Of the patients with functional obstruction who underwent endonasal surgical DCR, 9/11 (82\%) showed improvement or resolution compared to $5 / 7(71 \%)$ in the laser group.

A paper recently published by Delaney and Khooshabeh ${ }^{5}$ used a different term, acquired partial nasolacrimal obstruction, to describe essentially the same condition. Results from 50 lacrimal procedures carried out on their patients indicated that external DCR had a high success rate, with $90 \%$ of patients showing objective improvement and $84 \%$ subjective improvement at the time of tube removal. A retrospective study carried out by Sahlin and Rose ${ }^{6}$ considered the outcome of DCR in 25 patients who had patent tear ducts preoperatively. In all, $60 \%$ of these reported improved symptoms postoperatively with $50 \%$ rating the procedure as a success. Objective testing showed a normal lacrimal drainage capacity in three out of four patients who still had symptoms, suggesting an alternative cause for the symptoms.

\section{Conclusion}

Previous studies have shown that carefully assessed patients with functional nasolacrimal obstruction have excellent results with open or endonasal DCR surgery.

Our survey shows that most ophthalmologists are seeing patients with epiphora. The assessment currently performed would not pick up many patients with functional obstruction, so these patients cannot be offered DCR surgery with a high chance of success. We recommend a simple assessment of all patients with epiphora with an FDDT on arrival, a careful history and slit-lamp examination, syringing by a doctor and isotope scans and/or DCGs in equivocal cases.

\section{References}

1 Hurwitz JJ. 'Functional obstruction' of the lacrimal drainage passages. Chapter 20. In: Hurwitz JJ (ed). The Lacrimal System. Lippincott and Wilkins, Philadelphia, New York, 1996.

2 O'Donnell B, Shah R. Dacrocyctorhinostomy for epiphora in the presence of a patent lacrimal system. Clin Exp Ophthalmol 2001; 29: 27-29.

3 Zaidy FH, Symanski S, Olver JM. Endoscopic and external dacrocystorhinostomy in the management of functional epiphora. ESOPRS abstract 2001.

4 Moore WMH, Bentley CR, Olver J. Functional and anatomic results following two types of endoscopic endonasal dacrocystorhinostomy, surgical and Holmium laser. Ophthalmology 2002; 109: 1575-1582.

5 Delaney YM, Khooshabeh R. External dacrocystorhinostomy for the treatment of acquired partial nasolacrimal obstruction in adults. Br J Ophthalmol 2002; 86: 533-535.

6 Sahlin S, Rose GE. Lacrimal drainage capacity and symptomatic improvement after dacrocystorhinostomy in adults presenting with patent lacrimal drainage systems. Orbit 2001; 20(3): 173-179.

7 Conway ST. Evaluation and management of 'functional' nasolacrimal blockage: results of a survey of the American Society of Ophthalmic plastic and reconstructive surgery. Ophthalmic Plast Reconstr Surg 1994; 10(3): 185-188.

8 Wobig J. Editorial comment. Ophthalmic Plast Reconstr Surg 1994; 10(3): 188.

9 Guzek JP, Ching AS, Hoang TA, Dure-Smith P, Llaurao JG, Yau DC. Clinical and radiological lacrimal testing in patients with epiphora. Ophthalmology 1997; 104: 1875-1881.

10 Wearne MJ, Pitts J, Frank J, Rose GE. Comparison of dacrocystography and lacrimal scintigraphy in the diagnosis of functional nasolacrimal duct obstruction. Br J Ophthalmol 1999; 83: 1032-1035. 